RAD HAZU. MATEMATIČKE ZNANOSTI

Vol. $23=538$ (2019): 31-49

DOI: https://doi.org/10.21857/94kl4cxxjm

\title{
ONE-ALPHA WEIGHTED NETWORK DESCRIPTORS
}

\author{
TANja Vojković And Damir VukičEvić
}

\begin{abstract}
Complex networks are often used to model objects and their relations. Network descriptors are graph-theoretical invariants assigned to graphs that correspond to complex networks. Transmission and betweenness centrality are well known network descriptors and networkness and network surplus have been recently analyzed. All these four descriptors are based on the unrealistic assumption about equal communication between all vertices. Here, we amend this by assuming that vertices on the distance larger then one communicate less than those that are neighbors. We analyze network descriptors for all possible values of the factor $\alpha$ that measures reduction in the communication of the vertices that are not neighbors. We term these descriptors one-alpha descriptors and determine their extremal values.
\end{abstract}

\section{INTRODUCTION AND MOTIVATION}

Many complex systems in nature and society can be described in terms of complex networks capturing the web of connections among the units they are made of. So the study of complex networks pervades many fields of science, from chemistry [12], biochemistry [1,16] to communication theory [14].

Many aspects of complex networks are studied and in this paper we observe the network from the aspect of communication between the vertices. Key issues are of organizational nature: Are there any universal principles regarding the structural design and dynamic of communication in complex networks? Finding the answers to this question brings us to network descriptors $[5,15]$.

A complex network is represented by a simple connected graph $G=(V, E)$ and in this paper we use standard terminology of graph theory [7].

We will take a look at commonly used network descriptors, values that strive to describe the dynamic of information transfer in the network and define a variation of these descriptors. Extremal values for the already known

2010 Mathematics Subject Classification. 05C82, 05C35.

Key words and phrases. Network descriptors, complex networks, extremal graph theory. 
descriptors are thoroughly analyzed and in this paper we will explore the extremal values of our newly defined descriptors.

Two most important descriptors are betweenness centrality and transmission. Betweenness centrality is defined and studied in papers [10,11] and it can be efficiently calculated by the algorithm of Brandes [6]. It has been applied to indicate the importance of an individual node $[2,10]$, and its extremal values have been analyzed in paper [8].

DEFINITION 1.1. Let $G$ be a simple connected graph. Edge betweenness $b_{G}(u v)$ of edge $u v \in E(G)$ is

$$
b_{G}(u v)=\sum_{\{k, l\} \in\left(\begin{array}{c}
V \\
2
\end{array}\right)} \frac{s_{u v}^{k l}}{s^{k l}},
$$

where $s_{u v}^{k l}$ is the number of shortest paths between vertices $k$ and $l$ that pass through the edge uv and $s^{k l}$ is the total number of shortest paths between $k$ and $l$.

Definition 1.2. Let $G$ be a simple connected graph. Betweenness centrality $c_{G}(u)$ of vertex $u \in V(G)$ is the sum of edge betweennesses of all the edges incident to $u$, that is

$$
c_{G}(u)=\sum_{v \in N(u)} b(u v),
$$

where $N(u)$ is the set of neighbors of vertex $u$.

This kind of centrality is defined in paper [13] as Adjusted betweenness centrality. Note that betweenness centrality defined in this way is closely related to, but different from Freeman's betweenness centrality $b_{G}(u)$ [8], it holds

$$
b_{G}(u)=c_{G}(u)-n+1 .
$$

Betweenness centrality can be interpreted as the quantity of communication processed by vertex $u$ [18] in the network.

Definition 1.3. Let $G$ be a simple connected graph. Transmission $t_{G}(u)$ of vertex $u \in V(G)$ is

$$
t_{G}(u)=\sum_{v \in V} d(u, v),
$$

where $d(u, v)$ is the distance between vertices $u$ and $v$.

Observing the aspect of communication in complex network, transmission can be interpreted as the cost of the vertex $u$ to the network [18].

Besides betweenness centrality and transmission we will observe two more network descriptors, networkness and network surplus [18] which can be interpreted as ways of measuring "productivity" and "profitability" of vertex $u$. They are defined as follows. 
DeFINITION 1.4. Let $G$ be a simple connected graph. Networkness $\rho_{G}(u)$ of vertex $u \in V(G)$ is

$$
\rho_{G}(u)=\frac{c(u)}{t(u)} .
$$

Definition 1.5. Let $G$ be a simple connected graph. Network surplus $\nu_{G}(u)$ of vertex $u \in V(G)$ is

$$
\nu_{G}(u)=c(u)-t(u) .
$$

As most often will be clear which graph $G$ we are observing, we will use the denotations $c(u), t(u), \rho(u), \nu(u)$ instead of $c_{G}(u), t_{G}(u), \rho_{G}(u), \nu_{G}(u)$.

Now we will describe our motivation for defining a variation of these descriptors.

Note that interpretation of the betweenness centrality as the amount of information processed by the vertex $u$ assumes that the quantity of the information exchanged by any two vertices is equal. As this is not a case in real life networks we searched for a descriptor which amends this problem and gives a more realistic representation of the actual communication dynamic in the network. In the paper [3] we presented one way of fixing this by defining a generalized version of the observed descriptors, where the amount of communication was weighted by $d(u, v)^{\lambda}$, for $\lambda<0$, and in paper [4] by weighting the amount of communication by $\lambda^{d(u, v)}$, for $\lambda \in\langle 0,1\rangle$.

Here we consider network descriptors where the amount of communication between two non-neighbor vertices decreases proportional to $\alpha$, compared to the amount of communication between neighboring vertices, for some $\alpha \in$ $\langle 0,1\rangle$. This modification, unlike the ones in [3] and [4], takes in the account that neighboring vertices communicate much more than non-neighboring ones, which is of course true in vast majority of networks.

We proceed by defining one-alpha weighted descriptors, and then analyzing their extremal values in simple connected graphs.

\section{DEFINING ONE-ALPHA WEIGHTED DESCRIPTORS}

The definitions of one-alpha weighted descriptors for $\alpha \in\langle 0,1\rangle$ are as follows.

Definition 2.1. Let $G$ be a simple connected graph and $\alpha \in\langle 0,1\rangle$. Onealpha weighted transmission $t_{\alpha}(u)$ of vertex $u \in V(G)$ is

$$
t_{\alpha}(u)=d(u)+\sum_{v \notin N(u)} d(u, v) \alpha,
$$

where $d(u)$ is the degree of the vertex $u$ and $d(u, v)$ is the distance between vertices $u$ and $v$. 
Definition 2.2. Let $G$ be a simple connected graph and $\alpha \in\langle 0,1\rangle$. Onealpha weighted betweenness centrality $c_{\alpha}(u)$ of vertex $u \in V(G)$ is

$$
c_{\alpha}(u)=\sum_{v \in N(u)} \sum_{\{k, l\} \in\left(\begin{array}{l}
V \\
2
\end{array}\right)} \frac{s_{u v}^{k l}}{s^{k l}} \cdot \begin{cases}1, & \text { if } d(k, l)=1, \\
\alpha, & \text { if } d(k, l)>1 .\end{cases}
$$

One-alpha weighted networknes and one-alpha weighted network surplus are defined analogously with

$$
\begin{gathered}
\rho_{\alpha}(u)=\frac{c_{\alpha}(u)}{t_{\alpha}(u)}, \\
\nu_{\alpha}(u)=c_{\alpha}(u)-t_{\alpha}(u) .
\end{gathered}
$$

Note that by putting $\alpha=1$ we get standard definitions of transmission, betweenness centrality, networkness and network surplus $[8,18]$. From the definitions is clear that the range for $\alpha, \alpha \in\langle 0,1\rangle$ is chosen because of the assumption that distant vertices communicate less than the close ones.

By observing the values of each of these descriptors for all vertices in a given graph, we can define a minimal and a maximal value of each descriptor in the graph:

$$
\begin{aligned}
& m t_{\alpha}(G)=\min \left\{t_{\alpha}(u): u \in V(G)\right\}, \\
& M t_{\alpha}(G)=\max \left\{t_{\alpha}(u): u \in V(G)\right\}, \\
& m c_{\alpha}(G)=\min \left\{c_{\alpha}(u): u \in V(G)\right\}, \\
& M c_{\alpha}(G)=\max \left\{c_{\alpha}(u): u \in V(G)\right\}, \\
& m \rho_{\alpha}(G)=\min \left\{N_{\alpha}(u): u \in V(G)\right\}, \\
& M \rho_{\alpha}(G)=\max \left\{N_{\alpha}(u): u \in V(G)\right\}, \\
& m \nu_{\alpha}(G)=\min \left\{\nu_{\alpha}(u): u \in V(G)\right\}, \\
& M \nu_{\alpha}(G)=\max \left\{\nu_{\alpha}(u): u \in V(G)\right\} .
\end{aligned}
$$

The main goal of this paper is to find lower and upper bounds of these values and the graph for which they are obtained, for all $\alpha \in\langle 0,1\rangle$.

First we prove an analogous result to Lemma 2.1. in paper [3], as it will prove useful in our following proofs.

Lemma 2.3. For each graph $G$ it holds

$$
\sum_{u \in V} t_{\alpha}(u)=\sum_{u \in V} c_{\alpha}(u) .
$$

Proof. It holds

$$
\sum_{u \in V} t_{\alpha}(u)=\sum_{u \in V} d(u)+\sum_{u \in V} \sum_{v \notin N(u)} d(u, v) \alpha .
$$


Let us prove the sum of betweenness centrality of all the vertices in $G$ equals that. We have

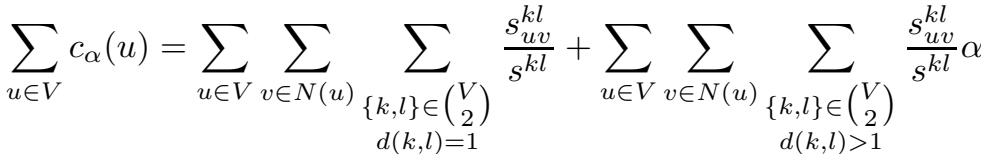

$$
\begin{aligned}
& =\sum_{\substack{\{k, l\} \in\left(\begin{array}{c}
V \\
2
\end{array}\right) \\
d(k, l)=1}} \frac{1}{s^{k l}} \sum_{u \in V} \sum_{v \in N(u)} s_{u v}^{k l}+\sum_{\substack{\{k, l\} \in\left(\begin{array}{c}
V \\
2
\end{array}\right) \\
d(k, l)>1}} \frac{1}{s^{k l}} \alpha \sum_{u \in V} \sum_{v \in N(u)} s_{u v}^{k l} .
\end{aligned}
$$

In the first sum, $s_{u v}^{k l}$ equals 1 only when $\{u, v\}=\{k, l\}$, and in other cases it equals $0 . s^{k l}$ always equals 1 since $k$ and $l$ are neighbors. So the first sum equals $\sum_{u \in V} d(u)$ because $\{u, v\}=\{k, l\}$ will hold when we observe $u$ and all its neighbors. In the second sum $s_{u v}^{k l}=s_{u v}^{l k}$ will equal 1 when $u v$ edge lies on the $k l$-path. Number of edges on the $k l$-path is $d(k, l)$, and there are $s^{k l}$ $k l$-paths so we have

$$
\begin{aligned}
& \sum_{\substack{\{k, l\} \in\left(\begin{array}{c}
V \\
2
\end{array}\right) \\
d(k, l)>1}} \frac{1}{s^{k l}} \alpha \sum_{u \in V} \sum_{v \in N(u)} s_{u v}^{k l}=\sum_{\substack{\{k, l\} \in\left(\begin{array}{c}
V \\
2 \\
d(k, l)>1
\end{array} \\
s^{k l}\right.}} \frac{\alpha}{d(k, d) \cdot s^{k l}} \\
& =\sum_{\substack{\{k, l\} \in\left(\begin{array}{c}
V \\
2 \\
d(k, l)>1
\end{array} \\
d\right.}} 2 \cdot \alpha \cdot d(k, l)=\sum_{u \in V} \sum_{v \notin N(u)} d(u, v) \alpha .
\end{aligned}
$$

It follows

$$
\sum_{u \in V} c_{\alpha}(u)=\sum_{u \in V} d(u)+\sum_{u \in V} \sum_{v \notin N(u)} d(u, v) \alpha,
$$

and the claim is proven.

REMARK 2.4. It is well-known that betweenness centrality and transmission are connected to Wiener index [19] defined by

$$
W(G)=\frac{1}{2} \sum_{(u, v) \in V^{2}} d(u, v)
$$

in the following way:

$$
\sum_{u \in V} t(u)=\sum_{u \in V} c(u)=2 W(G)
$$

So considering the result in Lemma 2.3 it is natural to denote

$$
W_{\alpha}(G)=\frac{1}{2} \sum_{u \in V} t_{\alpha}(u)=\frac{1}{2} \sum_{u \in V} c_{\alpha}(u) .
$$




\section{Results - LOWER AND UPPER BOUNDS}

3.1. One-alpha weighted transmission. In the proofs of our theorems we shall need the concept of Dijkstra tree [9]. Let $v$ be a vertex in a graph $G$. Dijkstra tree of vertex $v$ is a spanning tree that consists of the shortest paths starting in the vertex $v$. Note that there can be more than one Dijkstra tree of vertex $v$.

TheOREm 3.1. Let $G$ be a graph with $n$ vertices and $\alpha \in\langle 0,1\rangle$. It holds

$$
m t_{\alpha}(G) \geq\left\{\begin{array}{cc}
1+2 \alpha(n-2), & \alpha<\frac{1}{2} \\
n-1, & \alpha \geq \frac{1}{2} .
\end{array}\right.
$$

The equality holds for the center of a star in the case $\alpha \geq \frac{1}{2}$, and for any pendant vertex of a star otherwise.

Proof. From the definition of one-alpha weighted transmission it is clear that the values of $t_{\alpha}(u)$, for any vertex $u$ in graph $G$ will be the same for each vertex in the Dijkstra tree of vertex $u$. Therefore we may conclude that $G$ that minimizes $m t_{\alpha}(G)$ is a tree. Let $G$ be a tree with $n$ vertices, and $u$ a vertex for which minimal transmission is obtained. Let us observe a tree $G^{\prime}$ obtained from $G$ in the following way. All the vertices that are on a distance 3 or greater from $u$ we cut and move to any neighbor of $u$, so that the new distance from $u$ to those vertices is 2 . We obviously decreased the value of $t_{\alpha}(u)$ with each moved vertex. Let $k$ be the degree of $u$. In $G^{\prime}$ it holds $t_{\alpha}(u)=k+2 \alpha(n-1-k)$.

Now we define $f(k)=k+2 \alpha(n-1-k)$, where $k \in[1, n-1], n$ and $\alpha$ are predefined constants, and by observing its extremal values we obtain the result.

TheOREm 3.2. Let $G$ be a graph with $n$ vertices and $\alpha \in\langle 0,1\rangle$. It holds that

$$
M t_{\alpha}(G) \leq\left\{\begin{array}{cc}
n-1, & \alpha \leq \frac{2}{n+1} \\
1+\alpha \cdot \frac{n^{2}-n-2}{2}, & \alpha>\frac{2}{n+1}
\end{array}\right.
$$

The equality holds for the end-vertex of a path in the case $\alpha>\frac{2}{n+1}$, and for the center of a star otherwise.

Proof. As in the proof of Theorem 3.1, we may conclude $G$ that maximizes $t_{\alpha}(G)$ is a tree. Let $G$ be a tree and $v$ a vertex for which maximal transmission is obtained. Let us observe the longest path in $G$ that contains vertex $v$. If $v$ is the end-vertex than let us denote by $u$ the other end-vertex of that path, and if $v$ is not the end-vertex let us denote by $u$ the end of that 
path which is farther from $v$. If both end-vertices are on the same distance from $v$ let $u$ be any one of those end-vertices. Now, if $G$ contains a leaf $w$ such that $w \neq u$ and $d(v, w)>1$, let us observe a graph where that leaf is cut and moved so that its only neighbor is $u$. We continue this procedure until we put on that path all the vertices which are on distance greater than 1 from $v$. Let this path be of length $k$. In the obtained tree $G^{\prime}$ it holds

$$
n=k+d(v) \text {. }
$$

In each step of the above procedure we increased the distances from $v$ so the transmission $t_{\alpha}(v)$ is larger (or the same) in $G^{\prime}$ then in $G$. Now we have

$$
t_{\alpha}(v)=\alpha \cdot \sum_{i=2}^{k} i+(n-k)=\alpha \cdot \frac{k^{2}+k-2}{2}+n-k .
$$

Proceeding as in the proof of Theorem 3.1 and analyzing the corresponding function, we obtain the values from the claim.

For the next result, lower bound of $M t_{\alpha}$, we first need a technical lemma.

LEMmA 3.3. There is $n_{0} \in \mathbb{N}$ such that for each $d \in \mathbb{N}$ and $n>d$ the following implication holds

$$
d^{2}-2 d^{1.525} \leq n \leq d^{2}+1 \Longrightarrow \sqrt{n-1} \leq d \leq \sqrt{n}+n^{0.4} .
$$

ProOF. Let $n_{0}=\left\lceil 2^{1.525 / 0.1375}\right\rceil$. From $n>d$ if follows $n \leq d^{2}+1$ so it obviously holds $\sqrt{n-1} \leq d$ and the left inequality is proven. For the right inequality, let us assume the opposite, $d>\sqrt{n}+n^{0.4}$. It follows

$$
\left(\sqrt{n}+n^{0.4}\right)^{2}-2\left(\sqrt{n}+n^{0.4}\right)^{1.525}<d^{2}-2 d^{1.525} \leq n .
$$

We have

$$
\begin{aligned}
n & >\left(\sqrt{n}+n^{0.4}\right)^{2}-2\left(\sqrt{n}+n^{0.4}\right)^{1.525} \geq n+2 n^{0.9}-2^{2.525} n^{0.7625}= \\
& =n+2 n^{0.7625}\left(n^{0.1375}-2^{1.525}\right)>n, \text { for } n>n_{0} .
\end{aligned}
$$

We obtained a contradiction, so the claim is proven.

REMARK 3.4. By the results in [17] there exists sufficiently large $n_{0}$ so that for any $n \geq n_{0}$, there exists a $d$-regular graph with diameter 2 and $n$ vertices so that it holds $d^{2}-2 d^{1.525} \leq n \leq d^{2}+1$. We will denote such graph by $H_{n, d}$.

TheOREM 3.5. Let $G$ be a graph with $n$ vertices, and $\alpha \in\langle 0,1\rangle$. It holds

$$
\lim _{n \rightarrow \infty}\left(\frac{m M t_{\alpha}(n)}{\sqrt{n}}-\min \{\sqrt{n}, 2 \alpha(\sqrt{n}-1)+1\}\right)=0,
$$

where $m M t_{\alpha}(n)$ is minimal value of $M t_{\alpha}$ for graph with $n$ vertices. Equality holds for a complete graph when $\alpha \geq \frac{1}{2}$ and for $H_{n, d}$ when $\alpha<\frac{1}{2}$. 
ProOF. We will prove the claim for two cases separately, depending on $\alpha$.

1) Let $\alpha \geq \frac{1}{2}$. It is easy to see that minimal value of $M t_{\alpha}$ will be obtained for a complete graph. For $u \in V\left(K_{n}\right)$ it holds $t_{\alpha}(u)=n-1$. It follows

$$
\begin{aligned}
\lim _{n \rightarrow \infty}\left(\frac{m M t_{\alpha}(n)}{\sqrt{n}}-\sqrt{n}\right) & =\lim _{n \rightarrow \infty}\left(\frac{M t_{\alpha}\left(K_{n}\right)}{\sqrt{n}}-\sqrt{n}\right) \\
& =\lim _{n \rightarrow \infty}\left(\frac{n-1}{\sqrt{n}}-\sqrt{n}\right)=0 .
\end{aligned}
$$

2) Let $\alpha<\frac{1}{2}$. Now it holds $\min \{\sqrt{n}, 2 \alpha(\sqrt{n}-1)+1\}=2 \alpha(\sqrt{n}-1)+1$. We will first show that

$$
\frac{m M t_{\alpha}(n)}{\sqrt{n}}-(2 \alpha(\sqrt{n}-1)+1) \geq \frac{(1-2 \alpha)(\sqrt{n-1}-\sqrt{n})-3 \alpha}{\sqrt{n}},
$$

for $n \geq\left(\frac{1-2 \alpha}{\alpha}\right)^{2}$.

Let $G_{n}$ be a graph that minimizes the maximal value of $t_{\alpha}$ in the family of graphs with $n$ vertices and let $u_{n}$ be a vertex that maximizes $t_{\alpha}$ in $G_{n}$. Let $d(n)=d\left(u_{n}\right)$. If $d(n) \geq \sqrt{n-1}$, we say that $G_{n}$ is of type $A$, and otherwise, we say that $G_{n}$ is of type $B$. Let us define

$$
\begin{aligned}
& m M A t_{\alpha}(n)=\left\{\begin{array}{cc}
+\infty, & d(n)<\sqrt{n-1}, \\
m M t_{\alpha}(n) & d\left(u_{n}\right) \geq \sqrt{n-1},
\end{array}\right. \\
& m M B t_{\alpha}(n)=\left\{\begin{array}{cc}
m M t_{\alpha}(n), & d(n)<\sqrt{n-1}, \\
+\infty, & d\left(u_{n}\right) \geq \sqrt{n-1} .
\end{array}\right.
\end{aligned}
$$

Obviously,

$$
m M t_{\alpha}(n)=\min \left\{m M A t_{\alpha}(n), m M B t_{\alpha}(n)\right\} .
$$

First, let us prove that

$$
\frac{m M A t_{\alpha}(n)}{\sqrt{n}}-(2 \alpha(\sqrt{n}-1)+1) \geq \frac{(1-2 \alpha)(\sqrt{n-1}-\sqrt{n})-3 \alpha}{\sqrt{n}},
$$

for each $n$.

Suppose to the contrary that there is $n$ such that the opposite holds. Then, $G_{n}$ is of type $A$, i.e. it holds $d(n) \geq \sqrt{n-1}$. We have

$$
\begin{aligned}
& m M A t_{\alpha}(n) \geq d(n)+(n-d(n)-1) 2 \alpha \geq(n-1) 2 \alpha+(1-2 \alpha) d(n) \\
& \geq(n-1) 2 \alpha+(1-2 \alpha) \sqrt{n-1} .
\end{aligned}
$$

Let us denote $f_{1}(n)=(n-1) 2 \alpha+(1-2 \alpha) \sqrt{n-1}$, so we have $m M A t_{\alpha}(n) \geq$ $f_{1}(n)$ and from this we obtain a contradiction. Similarly, we prove

$$
\frac{m M B t_{\alpha}(n)}{\sqrt{n}}-(2 \alpha(\sqrt{n}-1)+1) \geq \frac{(1-2 \alpha)(\sqrt{n-1}-\sqrt{n})-3 \alpha}{\sqrt{n}},
$$


for each $n>\left(\frac{1+2 \alpha}{\alpha}\right)^{2}$.

By the results in [17] there exists sufficiently large $n_{0}$ so that for any $n \geq n_{0}$, there exists a $d$-regular graph with diameter 2 , and $n$ vertices and it holds $d^{2}-2 d^{1.525} \leq n \leq d^{2}+1$. Let $G_{n}$ be such a graph with $n$ vertices, and $d(n)$ the degree of its vertices.

It obviously holds

$$
M t_{\alpha}\left(G_{n}\right)=d(n)+(n-d(n)-1) 2 \alpha=(n-1) 2 \alpha+(1-2 \alpha) d(n) .
$$

For sufficiently large $n$, now we have

$$
\frac{(1-2 \alpha)(\sqrt{n-1}-\sqrt{n})-2 \alpha}{\sqrt{n}} \leq \frac{M t_{\alpha}\left(G_{n}\right)}{\sqrt{n}}-(2 \alpha(\sqrt{n}-1)+1) .
$$

For $G_{n}$ we have $n \leq d(n)^{2}+1$ and therefore $d(n) \geq \sqrt{n-1}$. We obtain

$$
\lim _{n \rightarrow \infty}\left(\frac{M t_{\alpha}\left(G_{n}\right)}{\sqrt{n}}-(2 \alpha(\sqrt{n}-1)+1)\right)=\lim _{n \rightarrow \infty}(1-2 \alpha)\left(\frac{d(n)}{\sqrt{n}}-1\right) .
$$

Now by using Lemma 3.3 and the Squeezing Theorem we prove

$$
\lim _{n \rightarrow \infty}\left(\frac{d(n)}{\sqrt{n}}-1\right)=0 .
$$

For the other side, we have

$$
\begin{aligned}
& \lim _{n \rightarrow \infty}\left(\frac{(1-2 \alpha)(\sqrt{n-1}-\sqrt{n})-2 \alpha}{\sqrt{n}}\right) \\
& =\lim _{n \rightarrow \infty}\left((1-2 \alpha)\left(\frac{\sqrt{n-1}}{\sqrt{n}}-1\right)-\frac{2 \alpha}{\sqrt{n}}\right)=0 .
\end{aligned}
$$

Now, by (3.1) and The Squeezing Theorem it follows

$$
\lim _{n \rightarrow \infty}\left(\frac{m M t_{\alpha}(n)}{\sqrt{n}}-(2 \alpha(\sqrt{n}-1)+1)\right)=0 .
$$

That completes the proof for the case 2) and the original claim is therefore proven.

Now we will observe the upper limit of $m t_{\alpha}$. First, we need two lemmas.

Lemma 3.6. Let $\alpha \in\langle 0,1\rangle, n \in \mathbb{N}, n \geq 2$. Let $S$ be a set of sequences $\left(x_{1}, x_{2}, \ldots, x_{\lfloor n / 2\rfloor}\right)$ such that $x_{1}+x_{2}+\ldots+x_{\lfloor n / 2\rfloor}=n-1$ and there exists $k \in\{1, \ldots,\lfloor n / 2\rfloor\}$ such that $x_{i} \geq 2$ for each $i<k$ and $x_{i}=0$ for each $i>k$. Let $S^{\prime}$ be the set of sequences of the form $\left(y_{1}, y_{2}, \ldots, y_{\lfloor n / 2\rfloor}\right)$ such that there is $k \in\{2, \ldots,\lfloor n / 2\rfloor\}$ such that $y_{k} \in\{0,1\}, y_{i}=2$ for each $2 \leq i<k$ and $y_{i}=0$ for each $i>k$. 
Let $T_{n}\left(x_{1}, x_{2}, \ldots, x_{\lfloor n / 2\rfloor}\right)$ be defined by

$$
T_{n}\left(x_{1}, x_{2}, \ldots, x_{\lfloor n / 2\rfloor}\right)=x_{1}+\sum_{i=2}^{\lfloor n / 2\rfloor} x_{i} \cdot i \cdot \alpha .
$$

Then it holds

$$
\max \left\{T_{n}(s): s \in S\right\}=\max \left\{T_{n}(s): s \in S^{\prime}\right\} .
$$

Proof. Suppose to the contrary. Let $\left(x_{1}, x_{2}, \ldots, x_{\lfloor n / 2\rfloor}\right) \notin S^{\prime}$ maximize $T_{n}$ in $S$. Then there is $k \geq 2$ such that $x_{k}>2$. Note that $k<\lfloor n / 2\rfloor$. Then,

$$
\left(x_{1}, x_{2}, \ldots, x_{k}-1, x_{k+1}+1, x_{k+2}, \ldots, x_{\lfloor n / 2\rfloor}\right) \in S .
$$

It follows that

$$
\begin{aligned}
& 0 \leq T_{n}\left(x_{1}, x_{2}, \ldots, x_{\lfloor n / 2\rfloor}\right)-T_{n}\left(x_{1}, x_{2}, \ldots, x_{k}-1, x_{k+1}+1, x_{k+2}, \ldots, x_{\lfloor n / 2\rfloor}\right) \\
& =k \cdot \alpha-(k+1) \cdot \alpha<-\alpha,
\end{aligned}
$$

which is a contradiction.

Lemma 3.7. Let $S^{\prime}$ be a set of sequences defined in Lemma 3.6. Then, the value $M_{n}=\max \left\{T_{n}(s): s \in S^{\prime}\right\}$ is:

i) $n-1$, for $\alpha<\frac{4}{3+n}$ and $n$ odd or $\alpha<\frac{4 n-12}{n^{2}-8}$ and $n$ even;

ii) $2+\frac{1}{4} \alpha \cdot\left(n^{2}-9\right)$, for $\alpha \geq \frac{4}{3+n}$ and $n$ odd;

iii) $2+\frac{1}{4} \alpha \cdot\left(n^{2}-8\right)$, for $\alpha \geq \frac{4 n-12}{n^{2}-8}$ and $n$ even.

Proof. If $n \leq 4$, the claim is obvious. Hence, let us assume $n \geq 5$. It can be easily seen that i) is obtained for the sequence $(n-1,0, \ldots, 0)$, ii) for the sequence $(2, \ldots, 2)$ and iii) for the sequence $(2, \ldots, 2,1)$. Moreover, it can be easily seen that $T_{n}(2, \ldots, 2)>T_{n}(n-1,0, \ldots, 0)$ if and only if $\alpha \geq \frac{4}{3+n}$, and that $T_{n}(2, \ldots, 2,1)>T_{n}(n-1,0, \ldots, 0)$ if and only if $\alpha \geq \frac{4 n-12}{n^{2}-8}$.

Hence, it is sufficient to prove that for all $n$ :

$$
M_{n} \leq \max \left\{n-1,2+\frac{1}{4} \alpha \cdot\left(n^{2}-9\right)\right\} .
$$

Let us distinguish two cases:

1) $n-x_{1}$ is odd.

Then,

$$
\begin{aligned}
& T_{n}\left(x_{1}, x_{2}, \ldots, x_{\lfloor n / 2\rfloor}\right) \\
& =x_{1}+\sum_{i=2}^{\frac{n-1-x_{1}}{2}+1} 2 \cdot i \cdot \alpha=-\frac{5 \alpha}{4}+\alpha n+\frac{\alpha n^{2}}{4}+x_{1}-\alpha x_{1}-\frac{\alpha n x_{1}}{2}+\frac{\alpha x_{1}^{2}}{4} .
\end{aligned}
$$

Simple calculation shows that the function corresponding to the above expression obtains maximal value in $f(2)$ or $f(n-1)$. It holds $f(2)=2+$ $\frac{1}{4} \alpha \cdot\left(n^{2}-9\right)$ and $f(n-1)=n-1$, so the case is proven. 
2) $n-x_{1}$ is even.

Note that in this case $x_{1} \neq n-1$. It holds:

$T_{n}\left(x_{1}, x_{2}, \ldots, x_{\lfloor n / 2\rfloor}\right)=-\alpha+\alpha n+\frac{\alpha n^{2}}{4}+x_{1}-\alpha x_{1}-\frac{\alpha n x_{1}}{2}+\frac{\alpha x_{1}^{2}}{4}$.

By similar reasoning as in the previous case and observing two subcases, depending whether $n$ is even or odd we obtain a contradiction with $n \geq 5$.

All the cases are analyzed so the claim is proven.

The upper bound of $m t_{\alpha}$ is solved for 2-connected graphs only and the general case remains an open problem.

THEOREM 3.8. Let $G$ be a 2-connected graph with $n$ vertices and $\alpha \in$ $\langle 0,1\rangle$. It holds

$$
m t_{\alpha}(G) \leq\left\{\begin{array}{rlrl}
2+\frac{1}{4} \alpha\left(n^{2}-9\right), & & \alpha \geq \frac{4}{3+n} \text { and } n \text { odd } \\
2+\frac{1}{4} \alpha\left(n^{2}-8\right), & \alpha \geq \frac{4 n-12}{n^{2}-8} \text { and } n \text { even } \\
n-1, & \alpha<\frac{4}{3+n} \text { and } n \text { odd or } \\
& \alpha<\frac{4 n-12}{n^{2}-8} \text { and } n \text { even } .
\end{array}\right.
$$

Equality holds for any vertex in a complete graph in the third case and for any vertex in a cycle in the first two cases.

Proof. Let $G$ be a 2-connected graph and $u$ any vertex in $G$. Let $v$ be the vertex farthest from $u$ and let $d(u, v)=D$. Since $G$ is 2 -connected it holds that for every $d<D$ there are at least 2 vertices on a distance $d$ from $u$. From that it is easily seen that $D \leq\left\lfloor\frac{n}{2}\right\rfloor$. Let us denote with $x_{i}$ the number of vertices on a distance $i$ from $u$, and let us observe the sequence $\left(x_{1}, \ldots, x_{\lfloor n / 2\rfloor}\right)$. This sequence is obviously in set $S$ defined in Lemma 3.6 and by Lemmas 3.6 and 3.7 it follows that the maximal one-alpha weighted transmission value of vertex $u$ is the one from the claim.

\subsection{One-alpha weighted betweenness centrality.}

TheOREm 3.9. Let $G$ be a graph with $n$ vertices and $\alpha \in\langle 0,1\rangle$. It holds

$$
m c_{\alpha}(G) \geq 1+(n-2) \alpha .
$$

The equality holds for any leaf in $G$.

Proof. Minimal one-alpha weighted betweenness centrality will be obtained for a tree because we observe the shortest paths between vertices. Let $G$ be a tree and $u$ any vertex in $V(G)$. Let $v$ be the farthest vertex from $u$. If $d(u)>1$ let us cut a branch from $u$ (not the one containing $v$ ), and connect 
it to vertex $v$. We made $c_{\alpha}(u)$ smaller. Now let us repeat the procedure until $d(u)=1$. It holds

$$
c_{\alpha}(u)=1+(n-2) \alpha .
$$

That is the minimal value of betweenness centrality in any graph with $n$ vertices because in the sum $\sum_{v \in N(u)} \sum_{\{k, l\} \in\left(\begin{array}{l}V \\ 2\end{array}\right)} \frac{s_{u v}^{k l}}{s^{k l}}, \frac{s_{u v}^{k l}}{s^{k l}}$ is always 1, and we count all the vertices in $G$ different from $u$ only once. So it follows

$$
1+(n-2) \alpha \leq m c_{\alpha}(G) .
$$

THEOREM 3.10. Let $G$ be a graph with $n$ vertices and $\alpha \in\langle 0,1\rangle$. It holds

$$
M c_{\alpha}(G) \leq(n-1)(1+\alpha(n-2)) .
$$

The equality holds for the center of a star.

Proof. The claim is proven similarly as in the Theorem 3.2, by moving the vertices in $G$ and increasing the value of betweenness centrality.

The upper bound of $m c_{\alpha}$ is given for 2-connected graphs only.

TheOREM 3.11. Let $G$ be a 2-connected graph with $n$ vertices and $\alpha \in$ $\langle 0,1\rangle$. It holds

$$
m c_{a}(G) \leq\left\{\begin{array}{cc}
2+\frac{1}{4} \alpha\left(n^{2}-9\right), & \alpha \geq \frac{4}{3+n} \text { and } n \text { odd } ; \\
2+\frac{1}{4} \alpha\left(n^{2}-8\right), & \alpha \geq \frac{4 n-12}{n^{2}-8} \text { and } n \text { even } ; \\
n-1, & \alpha<\frac{4}{3+n} \text { and } n \text { odd or } \\
& \alpha<\frac{4 n-12}{n^{2}-8} \text { and } n \text { even. }
\end{array} .\right.
$$

Equality holds for any vertex of a complete graph in the third case and for any vertex of a cycle in the first two cases.

Proof. By Lemma 2.3 we have

$$
\sum_{v \in V} t(v)=\sum_{v \in V} c(v)
$$

so the claim follows from the proof of Theorem 3.8.

REMARK 3.12. The lower bound of $M c_{\alpha}(G)$ is still an open problem. 
3.3. One-alpha weighted networkness. First we prove a claim which will be used in some of the proofs in this section.

LEMMA 3.13. Let $G$ be a graph with $n$ vertices and $u$ its vertex with the degree $D$. It holds

$$
t_{\alpha}(u) \leq D+\alpha \sum_{i=2}^{n-D} i
$$

The equality holds when all non-neighbors of $u$ are on a single path starting with one fixed neighbor of $u$.

Proof. The claim is easily proven by observing the Dijkstra's tree of graph $G$ and moving vertices as to obtain a broom, similarly as in the proof of Theorem 3.2.

Theorem 3.14. Let $G$ be a graph with $n$ vertices and $\alpha \in\langle 0,1\rangle$. It holds

$$
\frac{1+\alpha(n-2)}{1+\frac{1}{2} \alpha\left(n^{2}-n-2\right)} \leq m \rho_{\alpha}(G) \leq 1 .
$$

The upper equality holds for any vertex of a vertex-transitive graph and the lower equality for an end vertex of a path.

Proof. For any graph $G$, by using Lemma 2.3, for the upper bound we have

$$
m \rho_{\alpha}(G)=\min \left\{\frac{c_{\alpha}(u)}{t_{\alpha}(u)}: u \in V(G)\right\} \leq \frac{\frac{1}{n} \sum_{u \in V(G)} c_{\alpha}(u)}{\frac{1}{n} \sum_{u \in V(G)} t_{\alpha}(u)}=\frac{2 W_{\alpha}(G)}{2 W_{\alpha}(G)}=1 .
$$

Let us prove the lower bound. Let $G$ be a graph for which the minimum of $m \rho_{\alpha}(G)$ is obtained and $u$ its vertex for which $m \rho_{\alpha}(G)=\rho_{\alpha}(u)$. Let $d$ be the degree of vertex $u$. One-alpha weighted betweenness centrality cannot be smaller than $d+(n-1-d) \alpha$ since $u$ lies on every shortest path between itself any every other vertex in a graph, while by Lemma 3.13 one-alpha weighted transmission is maximized when all non-neighbors of $u$ are on a single path starting with some fixed neighbor of $u$. So it holds

$m \rho_{\alpha}(G)=\frac{c_{\alpha}(u)}{t_{\alpha}(u)} \geq \frac{d+(n-1-d) \alpha}{d+\alpha \sum_{i=2}^{n-d} i}=\frac{d+\alpha(n-d-1)}{d+\frac{1}{2} \alpha\left(d^{2}+n+n^{2}-2-d(1+2 n)\right)}$.

Hence, it remains to minimize the function of $d, f:[1, n-1] \rightarrow \mathbb{R}$ given by the above expression. Calculation shows that it achieves its minimum for $d=1$ so it follows that the minimum is obtained for an end-vertex of a path. 
Definition 3.15. Let $k_{1}, k_{2}, \ldots, k_{d}$ be non-negative integers. Thorny star $S\left(k_{1}, \ldots, k_{d}\right)$ is a tree $G$ with $n=k_{1}+\ldots+k_{d}+1$ vertices such that there exists a vertex $u$ with degree $d$ so that $d(u, v) \leq 2$, for each $v \in V(G) \backslash\{u\}$, and that $k_{1}, \ldots, k_{d}$ are numbers of vertices in the components of $G-u$.

The example of a thorny star is given in Figure 1.

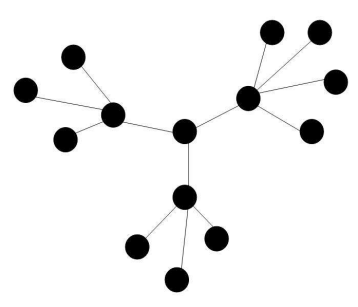

Figure 1. Thorny star $\mathrm{S}(4,5,4)$

Lemma 3.16. Let $x_{1}, x_{2}, \ldots, x_{d}$ be non-negative real numbers and $x_{1}+x_{2}+$ $\ldots+x_{d}=n-1$. It holds $\sum_{1 \leq i<j \leq d} x_{i} x_{j} \leq\left(\begin{array}{c}n-1 \\ 2\end{array}\right)-d\left(\frac{\frac{n-1}{d}}{2}\right)$.

Proof. Simple calculation shows that if $x_{1}=x_{2}=\ldots=x_{d}$ it follows $\sum_{1 \leq i<j \leq d} x_{i} x_{j}=\left(\begin{array}{c}n-1 \\ 2\end{array}\right)-d\left(\frac{\frac{n-1}{d}}{2}\right)$. Now let us assume $\left(y_{1}, \ldots, y_{d}\right)$ to be a $d$-tuple of non-negative real numbers such that the following stands

i) $y_{1}+y_{2}+\ldots+y_{d}=n-1$,

ii) $k, l \in\{1, \ldots, d\}$ exists such that $\left|y_{k}-y_{l}\right|>0$,

iii) $\left(y_{1}, \ldots, y_{d}\right)$ maximizes the sum $\sum_{1 \leq i<j \leq d} x_{i} x_{j}$.

Without the loss of generality let us assume that $k<l$ (otherwise we reverse their notation) and $y_{k}<y_{l}$. Let us denote $\sigma=\sum_{1 \leq i<j \leq d} y_{i} y_{j}$.

Now we observe a $d$-tuple

$$
\left(z_{1}, z_{2}, \ldots, z_{d}\right)=\left(y_{1}, \ldots, y_{k}+\varepsilon, \ldots, y_{l}-\varepsilon, \ldots, y_{d}\right),
$$

$\varepsilon=\frac{y_{l}-y_{k}}{2}$. Let us denote $\sigma^{\prime}=\sum_{1 \leq i<j \leq d} z_{i} z_{j}$. Obviously $z_{1}+\ldots+z_{d}=n-1$ still holds. It is easy to show that $\sigma^{\prime}=\sigma+2 \varepsilon\left(y_{l}-y_{k}-\varepsilon\right)$. Since $\varepsilon<y_{l}-y_{k}$, it holds $\sigma^{\prime}>\sigma$. This is in contradiction with iii), hence indeed $\left(\frac{n-1}{d}, \ldots, \frac{n-1}{d}\right)$ maximizes the sum $\sum_{1 \leq i<j \leq d} x_{i} x_{j}$. 
TheOREM 3.17. Let $G$ be a graph with $n$ vertices and $\alpha \in\langle 0,1\rangle$. It holds

$$
1 \leq M \rho_{\alpha}(G) \leq\left\{\begin{array}{cc}
\frac{4+\left(n^{2}-6\right) \alpha}{4(1+\alpha(n-3))}, & \text { if } 0<\alpha \leq \frac{n-7}{2 n^{2}-2 n} ; \\
\left\{\begin{array}{c}
q+(n-q-1) \alpha+ \\
\left.\left[\begin{array}{c}
n-1 \\
2
\end{array}\right)-q\left(\frac{n-1}{q}\right)\right] \cdot 2 \alpha
\end{array}\right\}, & \text { if } \frac{n-7}{2 n^{2}-2 n}<\alpha<\frac{n-3}{2(n-2)} \\
\frac{q+(n-1-q) \cdot 2 \alpha}{1+(n-2) \alpha,} & \text { if } \frac{n-3}{2(n-2)} \leq \alpha<1 ;
\end{array}\right.
$$

where

$$
\begin{aligned}
& q=\frac{1}{2(2-2 \alpha-n+2 \alpha n)}(2-4 \alpha-2 n+4 \alpha n- \\
& \left.\quad \sqrt{(-2+4 \alpha+2 n-4 \alpha n)^{2}-4(2-2 \alpha-n+2 \alpha n)\left(2 \alpha-4 \alpha n+2 \alpha n^{2}\right)}\right) .
\end{aligned}
$$

The lower equality holds for any vertex of a vertex-transitive graph and the upper equality for the central vertex of a graph $S\left(\frac{n-1}{2}, \frac{n-1}{2}\right)$ in the first case (when $n$ is odd), for the central vertex of a graph $S\left(\frac{n-1}{q}, \ldots, \frac{n-1}{q}\right)$ in the second case (when $q$ and $n / q$ are integers) and for the central vertex of a star in the third case.

Proof. The lower bound is easily proven as in the proof of Theorem 3.14. Let us prove the upper bound. First we observe that from Theorems 3.1 and 3.10 it follows that for $\alpha \geq \frac{1}{2}$ the equality will hold for the central vertex of a star, since that vertex has minimal transmission and maximal betweenness centrality value for $\alpha \geq \frac{1}{2}$. So let $\alpha<\frac{1}{2}, G$ be a graph with $n$ vertices and $u$ its vertex in which maximal value of one-alpha weighted networkness is obtained. As before we conclude that $G$ must be a tree. Let $d$ be the degree of vertex $u$ and let $k_{1}, \ldots, k_{d}$ be the number of vertices in components of $G-u$, respectively. Denote by $\Gamma\left(k_{1}, \ldots, k_{d}\right)$ family of all graphs $H$ that have vertex $u$ of degree $d$ and $k_{1}, \ldots, k_{d}$ vertices in components of $H-u$, respectively. Note that one-alpha weighted betweenness centrality of vertex $u$ in each such graph in $\Gamma\left(k_{1}, \ldots, k_{d}\right)$ is $d+(n-d-1) \alpha+\sum_{1 \leq i<j \leq d} k_{i} k_{j} \cdot 2 \alpha$. Hence, networkness of graph in $\Gamma\left(k_{1}, \ldots, k_{d}\right)$ is maximized when transmission is minimized, i.e. for thorny star $S\left(k_{1}, . ., k_{d}\right)$, therefore the upper bound is achieved for some $G=S\left(k_{1}, . ., k_{d}\right)$.

Now we have

$$
\rho_{\alpha}(u)=\frac{c_{\alpha}(u)}{t_{\alpha}(u)}=\frac{d+(n-d-1) \alpha+\sum_{1 \leq i<j \leq d} k_{i} k_{j} \cdot 2 \alpha}{d+(n-1-d) \cdot 2 \alpha} .
$$


From Lemma 3.16 we have $\sum_{1 \leq i<j \leq d} x_{i} x_{j} \leq\left(\begin{array}{c}n-1 \\ 2\end{array}\right)-d\left(\begin{array}{c}\frac{n-1}{d} \\ 2\end{array}\right)$, hence, it remains to maximize the function of $d, f:[1, n-1] \rightarrow \mathbb{R}$ given by the above expression Further calculation leads to the claim.

REMARK 3.18. Let us illustrate the case when graph $S\left(\frac{n-1}{d}, \ldots, \frac{n-1}{d}\right)$ from Theorem 3.17 is a tight upper bound. For $\alpha=\frac{1}{12}, n=10$ and $d=3$ the bound is reached for $S(3,3,3)$. See Figure 2 .

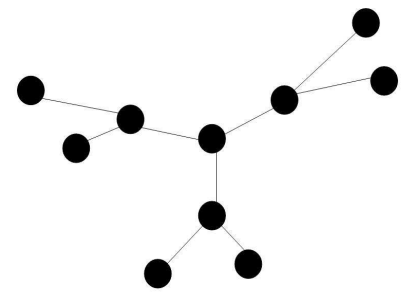

Figure 2. Thorny star $\mathrm{S}(3,3,3)$

3.4. Network surplus.

TheOREM 3.19. Let $G$ be a graph with $n$ vertices and $\alpha \in\langle 0,1\rangle$. It holds

$$
-\frac{n^{2}-3 n+2}{2} \alpha \leq m \nu_{\alpha}(G) \leq 0 \text {. }
$$

The lower equality holds for an end-vertex of a path and the upper equality for any vertex in a vertex-transitive graph.

Proof. The upper bound is easily proven as in the proof of Theorem 3.14. Let us prove the lower bound. Let $G$ be a graph with $n$ vertices and $u$ vertex that minimizes the value of one-alpha weighted network surplus. By similar reasoning as in Theorem 3.14 we have

$$
m \nu_{\alpha}(G)=c_{\alpha}(u)-t_{\alpha}(u)=-\frac{d^{2}+d-2 d n+n(n-1)}{2} \alpha,
$$

where $d$ is the degree of vertex $u$. The value of $\nu_{\alpha}$ for an end vertex of a path is $-\frac{n^{2}-3 n+2}{2} \alpha$ and with simple calculation we have

$$
-\frac{n^{2}-3 n+2}{2} \alpha-\left(-\frac{d^{2}+d-2 d n+n(n-1)}{2} \alpha\right)=\frac{(d-1)(d-2 n+2)}{2} \alpha .
$$

The right side is always negative, for any degree $d>1$, so we conclude that the lower bound is reached for an end-vertex of a path. 
Theorem 3.20. Let $G$ be a graph with $n$ vertices and $\alpha \in\langle 0,1\rangle$. It holds

$$
0 \leq M \nu_{\alpha}(G) \leq \alpha\left(n^{2}-3 n+2\right) .
$$

The lower equality holds for any vertex of a vertex-transitive graph and the upper equality for a central vertex of a star.

Proof. The lower bound is easily proven as in the proof of Theorem 3.14. Let us prove the upper bound. Let $G$ be a graph with $n$ vertices and $u$ vertex that maximizes the value of one-alpha weighted network surplus. By similar reasoning as in Theorem 3.17 we obtain

$$
M \nu_{\alpha}(G)=\frac{\alpha\left(d^{2}-(n-1)^{2}+d\left(n^{2}-3 n+2\right)\right)}{d},
$$

where $d$ is the degree of vertex $u$. The value of $\nu_{\alpha}$ for the central vertex of a star is $\alpha\left(n^{2}-3 n+2\right)$ and with simple calculation we have

$$
\alpha\left(n^{2}-3 n+2\right)-\frac{\alpha\left(d^{2}-(n-1)^{2}+d\left(n^{2}-3 n+2\right)\right)}{d}=\frac{\alpha\left((n-1)^{2}-d^{2}\right)}{d} .
$$

The right side is always positive, for any degree $d<n-1$, so we conclude that the upper bound is reached for the central vertex of a star.

\section{Conclusions}

In the view of fast growing field of complex network and communications theory, new, better ways to describe the behavior of information in the network are proposed all the time. Here we modified four well studied network descriptors, trying to implement the fact that vertices on distance one communicate much more then those on larger distances. For our modified descriptors, named one-alpha weighted network descriptors, we analyzed the extremal values and in each solved case found the numerical bound, the graph in which that value is obtained and the vertex for which it is obtained. Some bounds we proven only in the case of 2-connected graphs, and one of the bounds remains an open problem. We hope that here defined modified descriptors will inspire further study and new ways of observing the dynamics of networks.

ACKNOWLEDGements.

Partial support of the Croatian Ministry of Science, Education and Sports (Grant numbers 177-0000000-0884 and 037-0000000-2779) and EuroGIGA Collaborative Research Project GReGAS is greatly acknowledged.

\section{REFERENCES}

[1] R. Albert, Scale-free networks in cell biology, J. Cell Sci. 118 (2005), 4947-4957.

[2] J. M. Anthonisse, The Rush in a Graph, (1st edition), Mathematisch Centrum, Amsterdam, 1971.

[3] S. Antunović, T. Kokan, T. Vojković and D. Vukičević, Generalised network descriptors, Glas. Mat. Ser. III 48 (2013), 211-230. 
[4] S. Antunović, T. Kokan, T. Vojković and D. Vukičević, Exponential Generalised Network Descriptors, Adv. Math. Commun. 13 (2019), 405-420.

[5] A. L. Barabási, Linked: How Everything is Connected to Everything Else and What It Means, (1st edition), Persus Publishing, Cambridge, 2002.

[6] U. Brandes, A faster algorithm for betweenness centrality, J. Math. Sociol. 25 (2001), 163-177.

[7] B. Bollobás, Modern Graph Theory, (1st edition), Springer, New York, 1998.

[8] G. Caporossi, M. Paiva, D. Vukičević and M. Segatto, Centrality and Betweenness: Vertex and Edge decomposition of the Wiener Index, MATCH Commun. Math. Comput. Chem. 68 (2012), 293-302.

[9] T. H. Cormen, C. E. Leiserson, R. L. Rivest and C. Stein, Introduction to Algorithms, (2nd edition), MIT Press and McGraw-Hill, Cambridge, 2001.

[10] L. Freeman, A Set of Measures of Centrality Based on Betweenness, Sociometry 40 (1977), 35-41.

[11] L. Freeman, Centrality in social networks: Conceptual clarification, Soc. Networks 1 (1978), 215-239.

[12] P. De Leenheer, D. Angeli and E. D. Sontag, Monotone chemical reaction networks, J. Math. Chem. 41 (2007), 295-314.

[13] S. Majstorović and G. Caporossi, Bounds and relations involving adjusted centrality of the vertices of a tree, Graph. Combin. 31.6 (2015), 2319-2334.

[14] P. R. Monge and N. Contractor, Theories of Communication Networks, Oxford University Press, Oxford, 2003.

[15] M. E. J. Newman, Networks: An Introduction, (1st edition), Oxford University Press, Oxford, 2010

[16] N. D. Price, J. L. Reed, J. A. Papin, S. J. Wiback and B. O. Palsson, Network-based analysis of metabolic regulation in the human red blood cell, J. Theor. Biol. 225 (2003), 185-94.

[17] J. Šiagiova and J. Širan, Approaching the Moore bound for diameter two by Cayley graphs, J. Combin. Theory B 102 (2012), 470-473.

[18] D. Vukičević and G. Caporossi, Network descriptors based on betweenness centrality and transmission and their extremal values, Discrete Appl. Math. 161 (2013), 26782686.

[19] H. Wiener, Structural determination of paraffin boiling points, J. Am. Chem. Soc. 69 (1947), 17-20. 


\section{$(1, \alpha)$-težinski mrežni deskriptori}

\section{Tanja Vojković i Damir Vukičević}

SAŽETAK. Kompleksne mreže često koristimo za modeliranje objekata i njihovih odnosa, a mrežni deskriptori su invarijante pridružene grafovima koji odgovaraju kompleksnim mrežama. Transmisija (transmission) i međupoloženost (betweenness centrality) su dobro poznati u teoriji kompleksnih mreža, a vršna produktivnost (networkness) i vršna profitabilnost (network surplus) su nedavno definirani deskriptori. Sva četiri deskriptora su definirana s nerealnom pretpostavkom o jednakoj količini komunikacije svaka dva vrha u grafu. U ovom radu definiramo izmijenjene verzije ovih deskriptora, uz pretpostavku da vrhovi na udaljenosti većoj od 1 komuniciraju manje nego vrhovi koji su susjedni. Koeficijentom $\alpha$ izražavamo redukciju u komunikaciji nesusjednih vrhova te za sve moguće vrijednosti koeficijenta $\alpha \in\langle 0,1\rangle$ analiziramo ekstremalne vrijednosti ovih deskriptora.

Tanja Vojković

Department of Mathematics, Faculty of Science

University of Split

21000 Split, Croatia

E-mail: tanja@pmfst.hr

Damir Vukičević

Department of Mathematics, Faculty of Science

University of Split

21000 Split, Croatia

E-mail: vukicevi@pmfst.hr

Received: 26.2.2019.

Revised: 17.6.2019.

Accepted: 17.9.2019. 
